\title{
Influence of spin polarization on resistivity of a two-dimensional electron gas in Si MOSFET at metallic densities
}

\author{
I. Shlimak ${ }^{1}$ (a), A. Butenko ${ }^{1}$, D. I. Golosov ${ }^{1}$, K.-J. Friedland ${ }^{2}$, S. V. Kravchenko ${ }^{3}$ \\ 1 Jack and Pearl Resnick Institute of Advanced Technology, Department of Physics, Bar-Ilan University, Ramat-Gan \\ 52900, Israel \\ 2 Paul-Drude Institut für Festkörperelektronik, Hausvogteiplatz 5-7, 10117, Berlin, Germany \\ 3 Physics Department, Northeastern University, Boston, MA 02115, U.S.A.
}

PACS 72.25.Dc - Spin-polarized transport in semiconductors

PACS 73.40.Qv - Metal-insulator-semiconductor structures

PACS $71.30 .+\mathrm{h}-$ Metal-insulator transitions and other electronic transition $=\mathrm{s}$

\begin{abstract}
Positive magnetoresistance (PMR) of a silicon MOSFET in parallel magnetic fields $B$ has been measured at high electron densities $n \gg n_{\mathrm{c}}$ where $n_{\mathrm{c}}$ is the critical density of the metalinsulator transition (MIT). It turns out that the normalized PMR curves, $R(B) / R(0)$, merge together when the field is scaled according to $B / B_{\mathrm{c}}(n)$ where $B_{\mathrm{c}}$ is the field in which electrons become fully spin polarized. The values of $B_{\mathrm{c}}$ have been calculated from the simple equality between the Zeeman splitting energy and the Fermi energy taking into account the experimentally measured dependence of the spin susceptibility on the electron density. This extends the range of validity of the scaling all the way to a deeply metallic regime far away from MIT. The subsequent analysis of PMR for low $n \gtrsim n_{\mathrm{c}}$ demonstrated that the merging of the initial parts of curves can bee achieved only with taking into account the temperature dependence of $B_{\mathrm{c}}$. It is also shown that the shape of the PMR curves at strong magnetic fields is affected by a crossover from a purely two-dimensional (2D) electron transport to a regime where out-of-plane carrier motion becomes important (quasi-three-dimensional regime).
\end{abstract}

Introduction. - Following the experimental observation of the the metal-insulator transition (MIT) in twodimensional (2D) systems (for a review, see, for example, Ref. 1), the resistivity of various 2D systems, including silicon-based metal-oxide-semiconductor field-effect transistors (MOSFETs) and GaAs-based heterostructures, was measured in a parallel magnetic field in the vicinity of MIT 22 9. These studies were largely motivated by the still open question concerning the influence of the electron spin polarisation on the MIT in 2D (indeed, a parallel magnetic field lifts the spin degeneracy without otherwise influencing the electron motion).

It has been observed that on the metallic side of the MIT, where $n>n_{\mathrm{c}}$ (for Si MOSFETs, $n_{\mathrm{c}} \approx 0.8 \div 1.0$. $10^{11} \mathrm{~cm}^{-2}$, depending on the degree of disorder), the sample resistance $R$ increases with the parallel magnetic field, saturating above some characteristic value of the field, $B_{\mathrm{c}}$. This value is interpreted as the field corresponding to complete spin polarisation of the electrons. Possible mecha-

(a) E-mail: shlimai@biu.ac.il nisms of the influence of the spin polarisation on the resistance were discussed theoretically in Refs. 1, 10, 11.

It was also observed 6, 7, that once the resistivity and magnetic field are rescaled as $R(B, n) / R(0, n)$ and $B / B_{\mathrm{c}}(n)$, the PMR curves measured at different densities merge into one. This enables one to find the values of $B_{\mathrm{c}}$ for higher electron densities where the full spin polarisation cannot be experimentally achieved: $B_{\mathrm{c}}$ can be considered as a variable parameter, to be determined from the best merging. Below, we will use this prescription.

In turn, the dependence of $B_{\mathrm{c}}$ on electron density has attracted significant attention. In Ref. [7, it was shown that $B_{\mathrm{c}}$ is an almost linear function of $n$ vanishing at a sample-independent value $n=n_{\chi} \approx 0.8 \cdot 10^{11} \mathrm{~cm}^{-2}$. This tendency of $B_{\mathrm{c}}$ to vanish at a finite electron density raises the question about a possible magnetic instability in a strongly correlated 2DEG, resulting in a spontaneous spin polarisation at $n<n_{\chi}$. Then the 2DEG magnetic susceptibility $\chi$, which is proportional to the product $g^{*} m^{*}$ of the effective Landé $g$-factor and the renormalised effec- 
tive mass $m^{*}$, must be singular (divergent) at $n=n_{\chi}$. Interestingly, critical density of suggested ferromagnetic instability $n_{\chi}$ as found in Ref. [7] for a (100) Si-MOSFET 11 is close to $n_{\mathrm{c}}=0.8 \cdot 10^{11} \mathrm{~cm}^{-2}$, the latter corresponding to the MIT. On the other hand, according to Ref. [14, the observed gradual increase of the product $g^{*} m^{*}$ upon approaching $n_{\mathrm{c}}$ from the metallic side does not lead to an actual divergence of $\chi$ at $n_{\mathrm{c}}$. This disagreement is discussed in Refs. [15, 16]. Thus, the dependence $B_{\mathrm{c}}(n)$ near $n_{c}$ remains an open question in the problem of the influence of electron spin polarisation on MIT in 2D.

In the vicinity of $n_{\mathrm{c}}$, the correct experimental values of $B_{\mathrm{c}}$ can be obtained only via very low temperature measurements because $B_{\mathrm{c}}$ shows a strong temperature dependence [9]. Meanwhile, far from the MIT, at $n \gg n_{\mathrm{c}}$, the degree of the spin polarisation is almost temperature-independent. In this region, the value of $B_{\mathrm{c}}$ can be determined from the ratio between Zeeman splitting $\Delta \varepsilon_{\mathrm{Z}}=g^{*} \mu_{\mathrm{B}} B$ (where $\mu_{\mathrm{B}}$ is the Bohr magneton) and the Fermi energy $\varepsilon_{\mathrm{F}}=2 n\left(\pi \hbar^{2} / m^{*} \nu_{\mathrm{s}} \nu_{\mathrm{v}}\right)$, where $\nu_{\mathrm{s}}$ and $\nu_{\mathrm{v}}$ are the spin and valley degeneracy factors. As a result, the experimental values of $B_{\mathrm{c}}$ obtained at low temperatures correctly represent the $T=0$ case. At these high densities, the 2DEG physics should be described by a conventional Fermi liquid picture; it is therefore instructive to compare it to the results obtained at lower densities where the complications related to the proximity to the MIT become pronounced.

In this Letter, we report measurements of PMR at high electron densities $n \gg n_{c}$; the subsequent analysis allows for determination of $B_{c}(n)$ and for a meaningful comparison with the low-density case $n \gtrsim n_{c}$.

Experimental. - We measure the resistivity of a $\mathrm{Si}$ MOSFET sample in a magnetic field parallel to the surface and directed along the current. The slot-gate sample was similar to those described in our previous publications [17, 18. The electron density was controlled by the gate voltage $V_{\mathrm{G}}: n=1.24 \cdot 10^{11} \mathrm{~cm}^{-2}\left(V_{\mathrm{G}}-0.34 \mathrm{~V}\right)$. The PMR curves were measured at $T=0.3 \mathrm{~K}$ by the standard lockin technique.

The high sensitivity of our setup allows for measuring a weak PMR signal at high electron densities up to $n=$ $2.1 \cdot 10^{12} \mathrm{~cm}^{-2}$. The actual values of gate voltages applied (and hence the densities achieved) are restricted by the gate breakdown at high $V_{G}$.

The data used in Figs. 4 and 5 were obtained in an earlier measurement at $T=50 \mathrm{mK}$, partly reported in Ref. [7.

Results and discussion. - Fig. 1 shows the PMR curves $R(B) / R(0)$ measured at different values $V_{\mathrm{G}}$ and, hence, at different $n$. One can see that the PMR decreases rapidly with increasing density (the corresponding values

\footnotetext{
${ }^{1} \mathrm{~A}$ strong enhancement of $m^{*}$ with decreasing electron density $n$ was also observed in a (111) Si-MOSFET [12. Analysis of these data shows 13 that the behaviour of $m^{*}(n)$ is similar to that in a (100) Si-MOSFET.
}

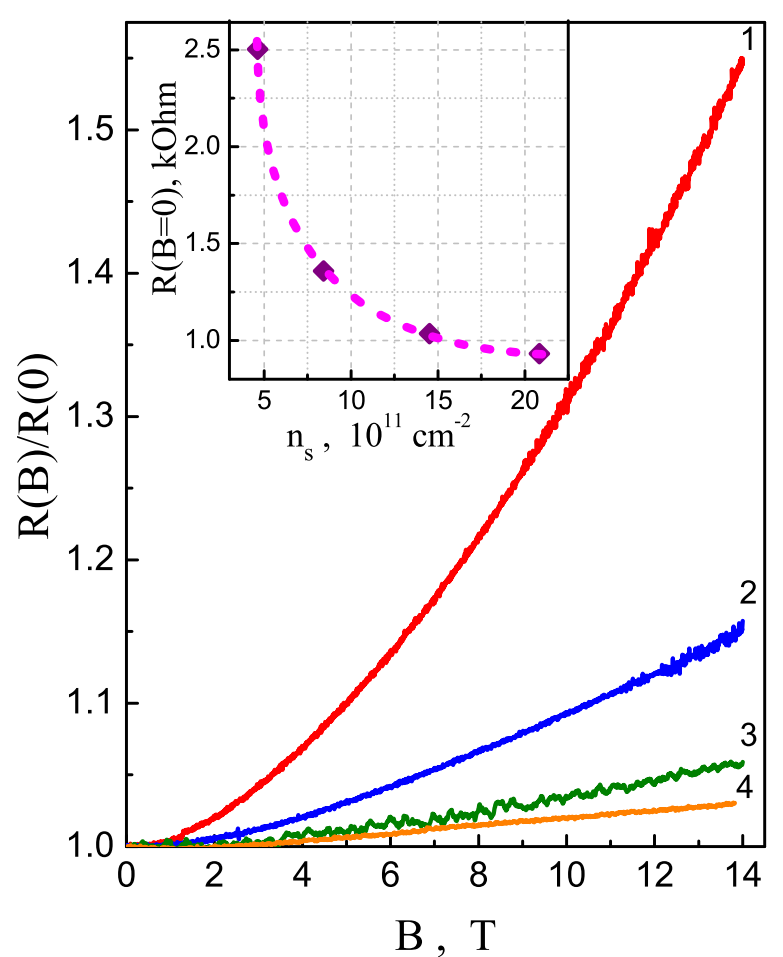

Fig. 1: (colour online) Normalised magnetoresistance $R(B) / R(0)$ of a Si MOSFET in a parallel magnetic field. Curves 1 to 4 correspond to gate voltages $V_{G}=4 \mathrm{~V}, V_{G}=7 \mathrm{~V}$, $V_{G}=12 \mathrm{~V}$, and $V_{G}=17 \mathrm{~V}$, respectively. Resistance $R(0)$ in the absence of the field is shown in the inset.

of $R(0)$ as a function of $n$ are shown in the inset to Fig. 1). The data allow us to calculate $B_{\mathrm{c}}$ from the condition that the Zeeman splitting and the Fermi energy are equal:

$$
B_{\mathrm{c}}=\frac{\pi \hbar^{2} n}{\left(g^{*} m^{*}\right) \mu_{\mathrm{B}}}
$$

When calculating the Fermi energy, it is taken into account that $\nu_{\mathrm{v}}=2$ for $\operatorname{Si}(100)$ MOSFET, and that $\nu_{\mathrm{s}}=1$ in a strong magnetic field. The other parameter that enters Eq. (10) is the renormalised value $\chi \propto\left(g^{*} m^{*}\right)$ which depends on the density. This latter dependence was reported in Ref. [14 in the dimensionless scale $\left(g^{*} m^{*}\right) / 2 m_{\mathrm{b}}$ vs. $r_{\mathrm{s}}$. Here, 2 is the bare $g$-factor for $\mathrm{Si}, m_{\mathrm{b}}=0.19 m_{0}$ the "bare" effective mass in $\mathrm{Si}(100)$, and $r_{\mathrm{s}}$ is the ratio between the Coulomb interaction energy and Fermi energy, $r_{\mathrm{s}}=m_{\mathrm{b}} e^{2} / 4 \pi \epsilon \epsilon_{0} \hbar(\pi n)^{1 / 2}$ ( with $\epsilon=7.7$ the dielectric constant of the interface between $\mathrm{Si}$ and $\mathrm{SiO}_{2}$, $\epsilon_{0}$ is vacuum permittivity). For Si MOSFET, one finds $r_{\mathrm{s}}=2.65 \cdot\left(10^{12} \mathrm{~cm}^{-2} / n\right)^{1 / 2}$. The calculated values of $B_{\mathrm{c}}$ are shown in Table 1

Because of the high electron densities, we are far from the saturation regime, and the values of $B_{\mathrm{c}}$ can be tested only by merging all the PMR curves together by scaling 
Table 1: Calculated values of $B_{c}$. The dependence of $g^{*} m^{*} / 2 m_{b}$ on $r_{s}$ is taken from Ref. [14].

\begin{tabular}{cccccc}
\hline No. & $V_{\mathrm{G}}, \mathrm{V}$ & $n, 10^{12} \mathrm{~cm}^{-2}$ & $r_{\mathrm{s}}$ & $g^{*} m^{*} / 2 m_{\mathrm{b}}$ & $B_{\mathrm{c}}, \mathrm{T}$ \\
\hline \hline 1 & 4 & 0.46 & 3.91 & 1.89 & 24.5 \\
2 & 7 & 0.84 & 2.89 & 1.64 & 41.4 \\
3 & 12 & 1.45 & 2.20 & 1.47 & 98.8 \\
4 & 17 & 2.08 & 1.84 & 1.38 & 151 \\
\hline
\end{tabular}

them with $B / B_{\mathrm{c}}$. Fig. 2 shows the result of this scaling. One can see that a good merging of all the curves supports our calculated values of $B_{\mathrm{c}}$. Finally, in Fig. 3 we plot the dependence of $B_{\mathrm{c}}(n)$. We note that at lower densities, the values of $B_{\mathrm{c}}(n)$ obtained from Eq. (1) are close to those determined directly from the saturation of the PMR at high fields [3, 7, 19].

We will now discuss the validity of the Fermi liquid-like approach which underlies Eq. (1) at these lower densities. Fig. 4 shows the PMR dependencies measured for the low electron densities $n \gtrsim n_{c}\left(1 \div 3 \cdot 10^{11} \mathrm{~cm}^{-2}\right)$ at $T=50 \mathrm{mK}$ and partly published in Ref. 7]. In Fig. 5. we plot these PMR curves $R(B) / R(0)$ as functions of $B / B_{\mathrm{c}}$, where $B_{\mathrm{c}}(n)$ is obtained from Eq. (1) with appropriately renormalised 14 values of $\left(g^{*} m^{*}\right)$. The result is shown in Fig 5 in the inset. We see that at the densities below $n=1.68 \cdot 10^{11} \mathrm{~cm}^{-2}$, the PMR curves at low fields deviate from the common scaled curve describing the higherdensity regime. We thus conclude that at these lower densities, our calculation of $B_{\mathrm{c}}(n)$ [see Eq. (1)] becomes inaccurate. Indeed, the data for $g^{*} m^{*}$ 14 used in our Fig. 3 were taken at $300 \mathrm{mK}$, while the MR curves were measured at $50 \mathrm{mK}$. It is known 9 that in the region near MIT the value of $B_{\mathrm{c}}$ (or equivalently $g^{*} m^{*}$ ) shows strong temperature dependence. Hence the data of Ref. [14 might not be appropriate for the analysis of our $R(B)$ curves at lower $n$, taken at about $50 \mathrm{mK}$. However, the higher-density regime, where Eq. (1) works perfectly, provides a reliable reference point for evaluating $B_{c}^{*}(n)$ from the requirement that the initial parts of all the $R\left(B / B_{c}^{*}\right) / R(0)$ collapse. The values of $B_{c}^{*}$, obtained in this way, are shown in Fig. 3 in the inset (dashed line). One sees that the values of $B_{c}^{*}$ indeed deviate from that of $B_{c}$ (solid line) at smaller $n$. The PMR curve merging is illustrated in Fig. [5 (main panel). One may conclude that for the lower concentrations, $n \lesssim 1.68 \cdot 10^{11} \mathrm{~cm}^{-2}$, the value of $k_{B} T$ with $T=300$ $\mathrm{mK}$ becomes smaller that the energy scale of the critical fluctuations, and therefore the values of $g^{*} m^{*}$ obtained in Ref. 14 no longer correspond to the true low-temperature limiting case (cf. Ref. [15]). Nevertheless, we note that in Ref. [14, the data for $g^{*} m^{*}$ were obtained from the analysis of Shubnikov-de Haas oscillations, whereas in our Fig.

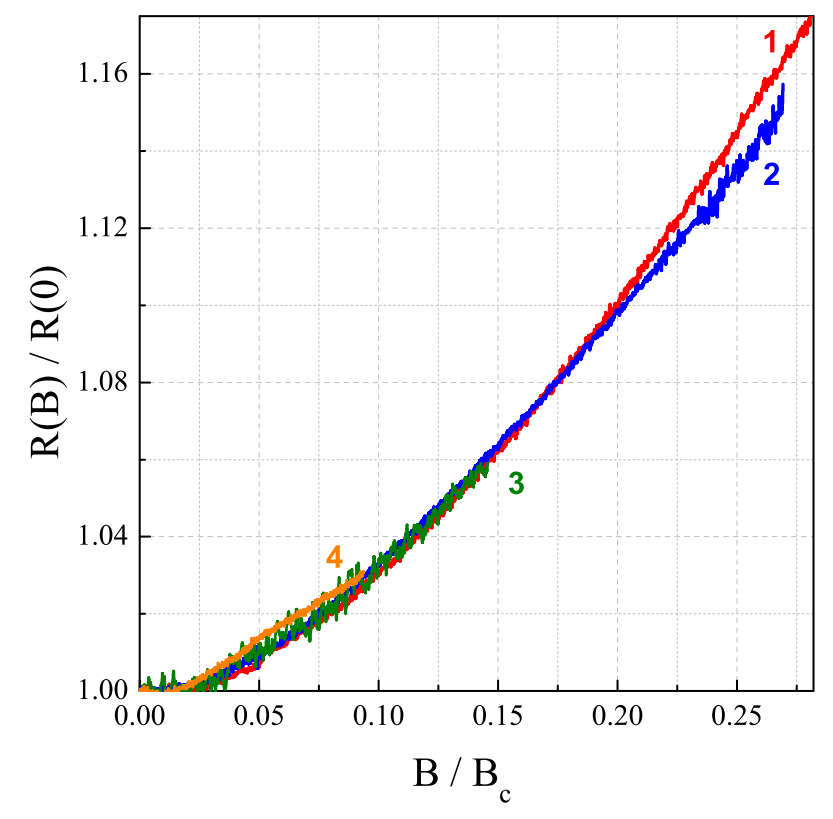

Fig. 2: (colour online) Normalised magnetoresistance rescaled as a function of $B / B_{c}$. Curves 1 to 4 correspond to gate voltages $4 \mathrm{~V}, 7 \mathrm{~V}, 12 \mathrm{~V}$, and $17 \mathrm{~V}$. The position of each curve number on the plot marks the end of the corresponding curve.

3 the values of $B_{c}^{*}$ (suggesting slightly different $g^{*} m^{*}$ ) were determined using an entirely different method, viz., the merging of all the MR curves. The absence of a precise agreement between the two is therefore not surprising.

It is seen from Fig. 4 that down to $n=1.68 \cdot 10^{11} \mathrm{~cm}^{-2}$, MR curves are saturated above $B=B_{\mathrm{c}}$, which shows that the value of $B_{\mathrm{c}}$ indeed corresponds to the complete spin polarisation. The behaviour at lower values of $n$ is somewhat different. We note that at $n<1.53 \cdot 10^{11} \mathrm{~cm}^{-2}$, the MR curves at $B=B_{\mathrm{c}}$ exhibit only a bend followed by a further increase of resistance with increasing magnetic field, and this behaviour becomes more pronounced with decreasing $n$. This feature is not related to the peculiar physics underlying the saturation of resistivity at $B=B_{\text {c }}$ as outlined above. Rather, we find that this further increase of resistance reflects a crossover from $2 \mathrm{D}$ to a quasi-3D mechanism of conductivity, due to the carrier motion in the out-of-plane direction which begins to play a role in a strong magnetic field, $B \gtrsim B_{2 D}$. At $B=B_{2 D}$, magnetic length $\lambda=(\hbar / e B)^{1 / 2}$ becomes comparable to the thickness of $2 \mathrm{D}$ layer $Z$, viz. $\lambda=\alpha Z$. Here, $\alpha$ is a pre-factor of the order of unity, to be determined phenomenologically; we find that $\alpha=0.4 \div 0.5$ yields a good fit (see the arrows in Fig. 4).

The values of $Z(n)$ (as estimated in Ref. [20]) and of $\lambda(B)$ are plotted in Fig. 6] (lower and upper curves, respectively). The dependence of $B_{2 D}$ on $n$ is shown in the inset. One can see that within the experimental interval of magnetic fields (up to $14 \mathrm{~T}$ ), conductivity of samples with higher $n$ always has a purely $2 \mathrm{D}$ character. However for smaller $n$, when the corresponding $B_{2 D}$ is below $14 \mathrm{~T}$, 


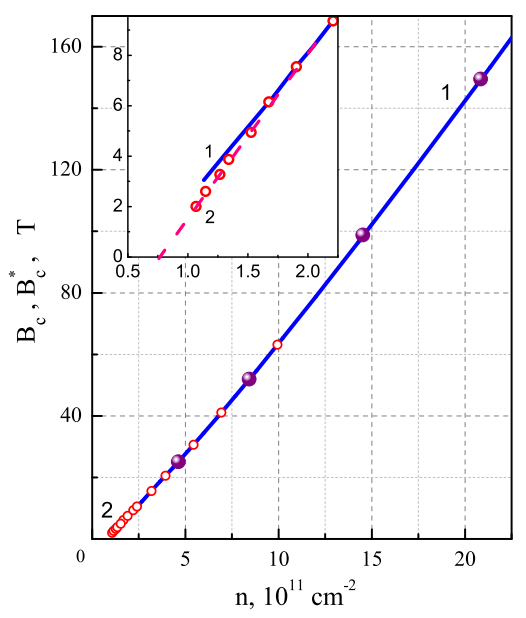

Fig. 3: (colour online) $B_{\mathrm{c}}$ as a function of $n$, Eq. (1), for high electron densities. Values given in Table 1 (and used in Fig. 2) are shown by bullets. The inset shows a blow-up of the low-density region.

the measured conductivity exhibits a transition from $2 \mathrm{D}$ to quasi-3D character with increase of $B$. This explains the absence of saturation and further increase of sample resistance $R$ after the bend in Fig. 4. Similar MR behaviour had been observed in bulk (3D) heavily doped $\mathrm{Si}$ with metallic conductivity [21].

Conclusion. - When viewed in the context of the earlier work on the lower-density Si MOSFETs, our results suggest the following generic conclusions:

(i) We have found that the PMR is present in the highdensity 2DEG far away from the MIT. In this region, the system is essentially a Fermi-liquid and its transport properties (including the PMR) are not directly related to the occurrence of the MIT at lower densities. This provides a further support to the theoretical studies [10,11] which obtain the PMR based on the Fermi liquid description. We speculate that a related magnetic field dependence would likely arise in the diffusion coefficient of a 2DEG without impurities.

(ii) We emphasise nevertheless that the PMR observed in the high-density regime must have the same underlying physics as the much stronger effect observed closer to the MIT. This is unequivocally illustrated by the fact that the same dimensionless scaling (in terms of $B / B_{c}(n)$ ) works throughout both regimes, which connect seamlessly. The scaling reflects a Fermi liquid-type renormalisation of the effective 2DEG parameters (in this case, $g^{*}$ and $m^{*}$ ). This suggests that the Fermi-liquid phenomenology itself is valid all the way down to the vicinity of the MIT, as long as the system remains in the ballistic regime. At yet lower densities, in the diffusive regime, the non-trivial interplay between the interactions and disorder, much discussed in the literature (see, e.g., Ref. 22]), strongly affects the Fermi liquid parameters, resulting in a strong increase of the spin susceptibility with decreasing temper-

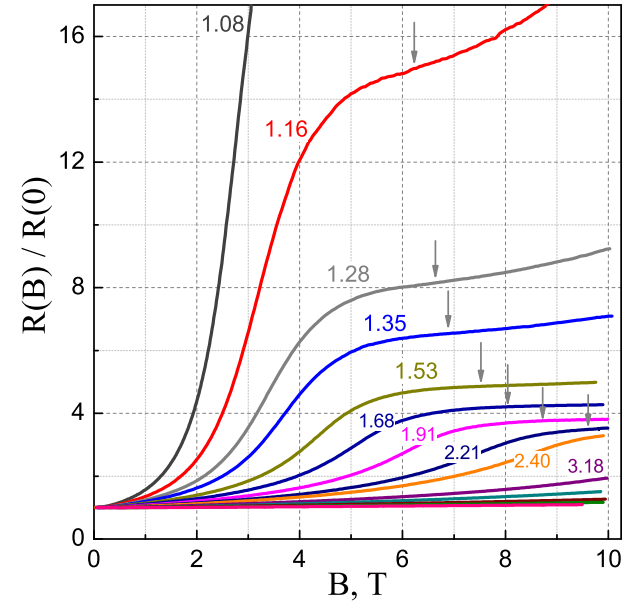

Fig. 4: (colour online) Magnetic field dependence of normalised magnetoresistance in the region of lower carrier densities (marked in the units of $10^{11} \mathrm{~cm}^{-2}$ ). Arrows denote the respective values of $B_{2 D}$ calculated for $\alpha=0.45$ as discussed in the text.

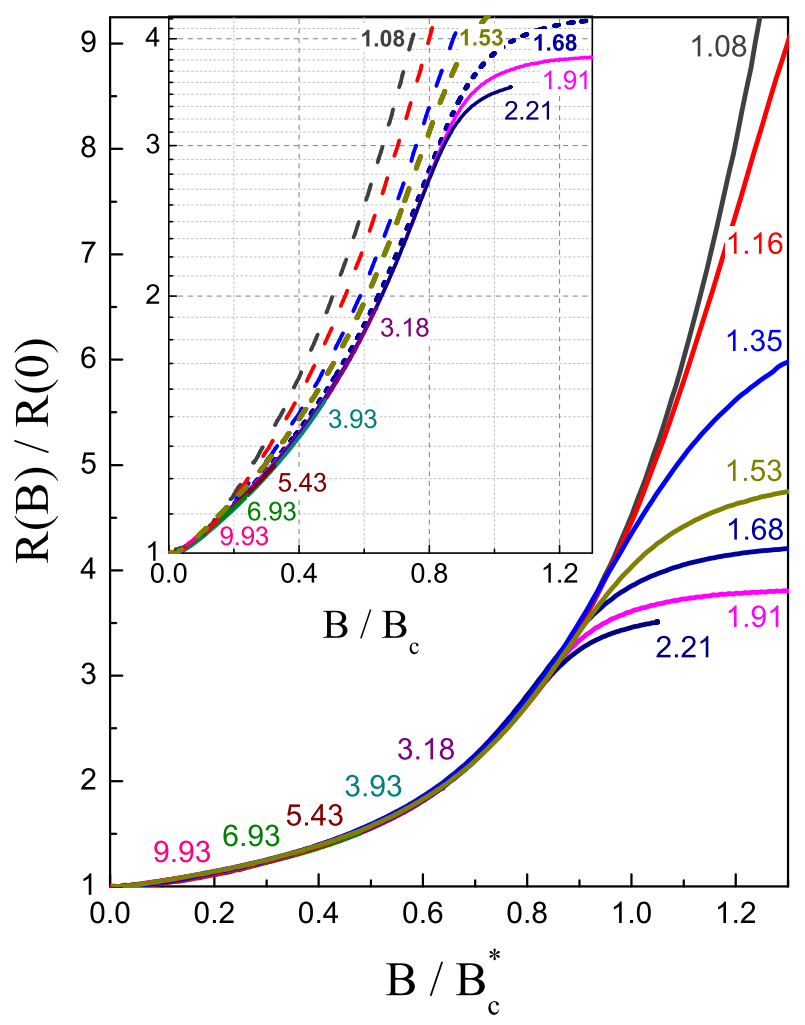

Fig. 5: (colour online) Normalised magnetoresistance $R(B) / R(0)$ scaled as a function of $B / B_{\mathrm{c}}$ (inset) and as a function of $B / B_{\mathrm{c}}^{*}$ (main panel). Here, $B_{\mathrm{c}}$ is given by eq. (1). and $B_{\mathrm{c}}^{*}$ is discussed in the text. Values of electron density $n$ are in units of $10^{11} \mathrm{~cm}^{-2}$; for $n \geq 2.21 \mathrm{~cm}^{-2}$, the position of each value on the plot marks the end of the corresponding curve. 


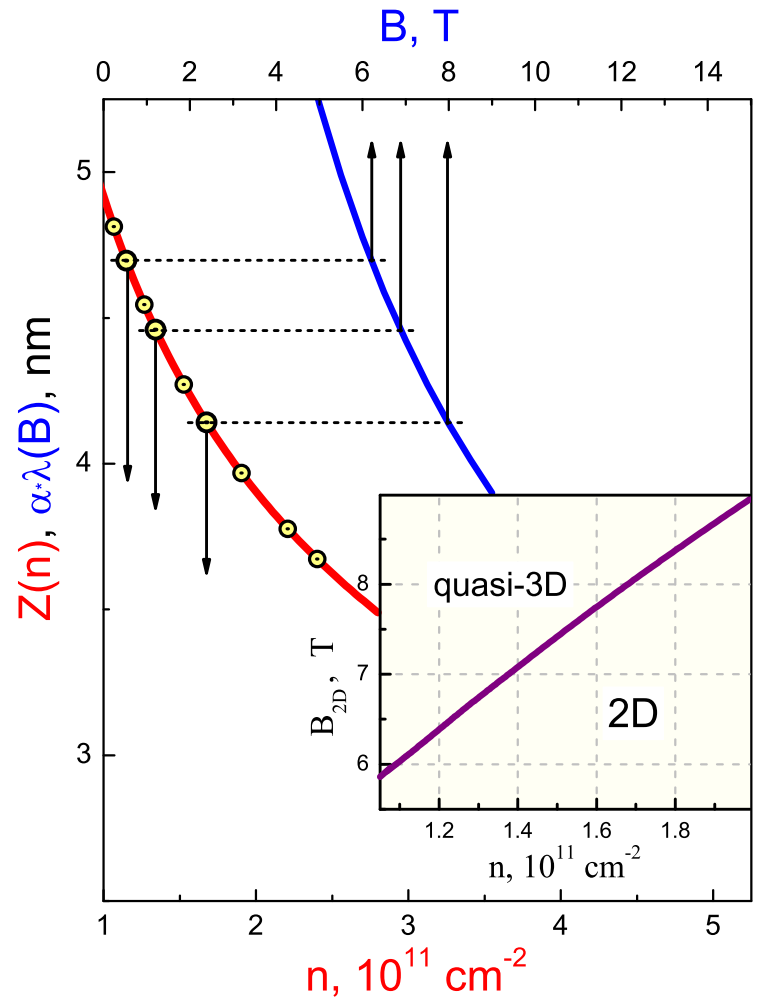

Fig. 6: (colour online) The dependence of conducting layer thickness $Z$ on the electron density $n$ (lower scale), and the scaled magnetic length $\alpha \lambda$ (for $\alpha=0.45$ ) as a function of the magnetic field (upper scale). The resultant dependence of $B_{2 D}$ on $n$ is shown in the inset.

ature, but this is beyond the scope of the present paper.

(iii) We find that with an increase of the parallel magnetic field $B$ beyond a certain value $B_{2 D}$ at a fixed $n$, the character of conductivity undergoes a crossover from twodimensional to a quasi-three-dimensional one when the magnetic length $\lambda$ becomes small in comparison to the layer width $Z$. This is directly observed in the regime of smaller densities $n$, where the value of $B_{2 D}$ lies within the experimentally studied range of $B$ up to $14 \mathrm{~T}$. The quasi$3 \mathrm{D}$ behaviour at high fields is reflected in a continued increase of sample resistance with $B$ instead of saturation.

We take pleasure in thanking A. Belostotsky and T. Hasenfratz for assistance, and R. Berkovits for helpful discussions. This work was supported in part by the BSF Grant 2006375 and by the Israeli Ministry of Immigrant Absorption. SVK was supported by DOE Grant DEFG02-84ER45153. IS thanks the Erick and Sheila Samson Chair of Semiconducting Technology for financial support.

\section{REFERENCES}

[1] Spivak B., Kravchenko S. V., Kivelson S. A. and
Gao X. P. A., Rev. Mod. Phys., 8220101743.

[2] Simonian D., Kravchenko S. V., Sarachik M. P. and Pudalov V. M., Phys. Rev. Lett., 7919972304.

[3] Pudalov V. M., Brunthaler G., Prinz A. and Bauer G., JETP Lett., 651997932.

[4] Oкамото T., Hosoya K., Kawaji S. and Yagi A., Phys. Rev. Lett., 8219993875.

[5] Mertes K. M., Simonian D., Sarachik M. P., Kravchenko S. V. and KlapwiJk T. M., Phys. Rev. B, 601999 R5093.

[6] Vitkalov S. A., Zheng H., Mertes K. M., SarachiK M. P. and KlapwiJk T. M., Phys. Rev. Lett., 872001 086401.

[7] Shashinin A. A., Kravchenko S. V., Dolgopolov V. T. and KlapwiJk T. M., Phys. Rev. Lett., 872001 086801.

[8] Shashkin A. A., Deviatov E. V., Dolgopolov V. T., Kapustin A. A., Anissimova S., Venkatesan A., Kravchenko S. V. and KlapwiJk T. M., Phys. Rev. B, 732006115420.

[9] Gao X. P. A., Boebinger G. S., Mills A. P. Jr., Ramirez A. P., Pfeiffer L. N. and West K. W., Phys. Rev. B, $732006241315(\mathrm{R})$.

[10] Dolgopolov V. T. and Gold A., JETP Lett., 712000 27.

[11] Zala G., Narozhny B. N. and Aleiner I. L. , Phys. Rev. B, 652001 020201(R).

[12] Shashinin A. A., Kapustin A. A., Deviatov E. V., Dolgopolov V. T. and Kwon Z. D., Phys. Rev. B, 76 2007 241302(R).

[13] Gold A. and Dolgopolov V. T., JETP Lett., 862007 600.

[14] Pudalov V. M., Gershenson M. E., Kojima H., Butch N., Dizhur E. M., Brunthaler G., Prinz A. and Bauer G., Phys. Rev. Lett., 882002196404.

[15] Kravchenko S. V., Shashin A. A. and Dolgopolov V. T., Phys. Rev. Lett., 892002219701.

[16] Fleury G. and Waintal X., Phys. Rev. B, 812010 165117.

[17] Shlimak I., Ginodman V., Butenko A., Friedland K.-J. and Kravchenko S. V., Europhys. Lett., 822008 47001.

[18] Shlimak I., Golosov D. I., Butenko A., Friedland K.-J. and Kravchenko S. V., Ann. Phys. (Berlin), 18 2009913.

[19] Broto J. M., Goiran M., Rakoto H., Gold A and Dolgopolov V. T., Phys. Rev. B, 672003161304.

[20] Ando T., Fowler A. B. and Stern F., Rev. Mod. Phys., 541982437.

[21] Shafarman W. N., Gastner T. G., Brooks J. S.,Martin K. P. and Naughton M. J., Solid-State Electronics, 28198593.

[22] Punnoose A. and Finkel'stein A. M., Science, 310 2005289. 